\title{
Fractional Derivative Reconstruction of Forced Oscillators*
}

\author{
G. Lin \\ University of Maryland \\ Department of Electrical and Computer Engineering \\ University Park, MD 20742 USA \\ B. F. Feeny \\ Michigan State University \\ Department of Mechanical Engineering \\ East Lansing, MI 48824 USA \\ T. Das \\ Michigan State University \\ Department of Mechanical Engineering \\ East Lansing, MI 48824 USA
}

\begin{abstract}
Fractional derivatives are applied in the reconstruction, from a single observable, of the dynamics of a Duffing oscillator and a two-well experiment. The fractional derivatives of time series data are obtained in the frequency domain. The derivative fraction is evaluated using the average mutual information between the observable and its fractional derivative. The ability of this reconstruction method to unfold the data is assessed by the method of global false nearest neighbors. The reconstructed data is used to compute recurrences and fractal dimensions. The reconstruction is compared to the true phase space and the delay reconstruction in order to assess the reconstruction parameters and the quality of results.
\end{abstract}

Keywords: phase-space reconstructions; fractional derivatives; fractional calculus; chaos; embeddings

\section{Introduction}

In this paper, we use fractional derivatives for the purpose of phase-space reconstructions of data from forced nonlinear oscillators.

The most common method of phase-space reconstruction is the method of delays $[1$, $2]$. Another reconstruction idea has been to use derivatives of the sampled quantity $[1,2]$. Takens has proven that both methods will produce embeddings, provided that the system is sufficiently smooth and noise free (see also Noakes [3]). However, real systems are not

*Nonlinear Dynamics 55 (3) 239-250. 
noise-free. The successive derivatives of the observed state are prone to increasing noise amplification as the order of the derivative increases.

In the method of delays, for a single observable $x_{j}, d$-dimensional pseudo vectors $\mathbf{y}_{j}$ are built with elements being the sampled observable separated by a constant delay time, such that $\mathbf{y}_{j}=\left[x_{j}, x_{j+h}, \cdots, x_{j+h(d-1)}\right]$, where $h$ is the delay index, and $d$ is embedding dimension, both of which are to be determined. The delay $h$ is chosen to make the components of $\mathbf{y}_{j}$ as independent as possible ( $h$ not too small), yet still deterministically related ( $h$ not too large). An accepted way to get the optimal value of $h$ is to choose the first minimum (if it exists) of the average mutual information $\bar{I}$ between $x_{j}$ and $x_{j+h}$ [4] for various values of $h$. (The independence between $x_{j}$ and $x_{j+n h}$, with $2<n<d-1$, which represents other pairs of axes in the delay reconstruction, is not addressed in the determination of the optimal choice of $h$.) Also, while there is an optimal $h$ with respect to the average mutual information computation, non optimal values of $h$ can still lead to phase-space reconstructions that represent the dynamics rather well, as long as the value of $h$ does not get into the aforementioned "too large" and "too small" categories. If the average mutual information does not achieve a minimum, some other criterion must be involved in the $h$ decision.

For an embedding, $d=d_{E}$ can be chosen, for example, by the method of false nearest neighbors (FNNs) [5], by which erroneous foldings of the sampled trajectories are detected as they unfold when the reconstruction dimension is incremented, or the method of Cao [6]. By the FNN method, $d_{E}$ is the minimum embedding dimension which has no (or few) erroneous foldings.

Distortions of the method of delays have been documented, for example by Potopov [7] and Mindlin [8]. An inherent problem is that any point in the embedding is represented by a finite time interval, $\left[t, t+\left(d_{E}-1\right) \tau\right]$, as opposed to an instant of time in the true phase space. In contrast, the derivatives method of reconstructions establishes reconstruction vectors that truly correspond to instants in time.

As an alternative to delay and derivative phase-space reconstructions, a mix of integrals and derivatives has been suggested $[9,10]$. The presence of an integral can introduce a drift in the reconstruction. Gilmore and Lefranc [10] suggest removing the mean of the signal to avoid such secular behavior, or incorporating history into the integral. High-pass filtering might also be an option [11]. Fractional deriviatives were used to reconstruct the dynamics of a Lorenz attractor [12].

In this work, we use fractional derivatives to reconstruct the dynamics of numerical and experimental forced oscillators. Average mutual information calculations suggest that fractional derivatives produce independent coordinates in the reconstructed phase space. Also, a fractional derivative can be incremented several times before accumulating a large order to the total derivative. Thus, it is possible to obtain moderate dimensions in the reconstructed phase spaces of low-noise systems without excessive amplification of the noise. The main task here is to illustrate the application of this idea to low-dimensional, forced oscillator simulation and experiment. This is distinguished from [12] by addressing non-autonomous systems in $\mathbf{R}^{2} \times \mathbf{S}^{1}$ space, with application to a real experiment. While the method stands up to these examples, a rigorous mathematical justification is currently absent. 


\section{Fractional Derivatives}

\subsection{Background}

Example applications of fractional derivatives include visco-elasticity models in oscillators [13-15], flows through porous media [16], and the characterization of fractal functions [17]. In the modeling examples, fractional derivatives appear as terms in the differential equations of motion, or equivalently as fractional power terms in the denominator of a transfer function. Recent special issues contain many applications of fractional derivatives in dynamical systems, and methods of analysis [18, 19].

The Liouville-Riemann fractional derivative can be expressed for non-integer order $a<0$ as

$$
\frac{d^{a} x(t)}{d\left(t-t_{0}\right)^{a}}=\frac{1}{\Gamma(-a)} \int_{t_{0}}^{t} \frac{x(\tau)}{(t-\tau)^{a+1}} d \tau .
$$

For $a>0$, one can apply

$$
\frac{d^{a} x(t)}{d\left(t-t_{0}\right)^{a}}=\frac{d^{n}}{d t^{n}} \frac{1}{\Gamma(n-a)} \int_{t_{0}}^{t} \frac{x(\tau)}{(t-\tau)^{a-n+1}} d \tau,
$$

for $n>a[20]$. The $t_{0}$ is often chosen as zero.

The fractional derivative is difficult to interpret in a spatial domain (in comparison to the familiar slope and curvature) and in the time domain (in comparison to velocity, acceleration, and jerk). In fact, the integral definitions imply that fractional derivatives have history dependence. However, it turns out that [20]

$$
\frac{d^{a} x(t)}{d\left(t-t_{0}\right)^{a}} e^{i \omega t} \rightarrow(i \omega)^{a} e^{i \omega t}, \quad t_{0} \rightarrow-\infty .
$$

Hence, at steady state, we interpret derivatives of dynamic signals, with sinusoidal elements, in the frequency domain based on a scaling of the amplitude by a fractional power of the frequency, and a shifting of the phase by a fraction of $\pi / 2$.

Thus in this work we compute the fractional derivative $d^{a} x(t) / d t^{a}$ of fractional order $a$ of a signal $x(t)$ by taking the fast Fourier transform (FFT) of the signal, multiplying by $(i \omega)^{a}$, and then transforming back to the time domain [21]. This is reminiscent of the similar "generalized derivative," obtained by exchanging the real and imaginary parts of the Fourier coefficients and multiplying by $|\omega|^{a}$ in the frequency domain, which was used to make two-dimensional plots that resembled a two-dimensional phase portrait [10].

Since the FFT has some approximation built in, we will refer to the computed fractional derivative of a signal $x(t)$ as $D^{a} x(t)$, in lieu of the true fractional derivative $d^{a} x(t) / d t^{a}$.

The FFT comes with its own problems, one of which is leakage, which leads to transient distortions at the endpoints of the the differentiated signal. To avoid geometric distortions, we do not apply the usual windowing to fix the leakage effects [22], but instead truncate the ends of the signal [12]. Another idea is to select a maximal subset of data for which the endpoints nearly match $[10]$. 


\subsection{Phase-Space Reconstruction}

We summarize the steps for reconstructing the phase space, of time series data $x\left(t_{n}\right)$ with a constant sampling rate, with fractional derivatives [12].

First, we have some computations that need be performed once:

1. Use the FFT of $x\left(t_{n}\right)$ to obtain the data $X\left(\omega_{n}\right)$ in the frequency domain.

2. Evaluate the post FFT leakage error by computing $\delta\left(t_{n}\right)=\left|D x\left(t_{n}\right)-\dot{x}\left(t_{n}\right)\right|$, where $D x\left(t_{n}\right)$ is obtained from the inverse FFT of $i \omega_{n} X\left(\omega_{n}\right)$ and $\dot{x}\left(t_{n}\right)$ is obtained by finite differences.

3. Find the interior range of samples for which $\delta\left(t_{n}\right)<\epsilon$, for some suitable $\epsilon$, to guide the truncation of the fractal derivative signals. This idea should work as long as $\delta$ is larger than the noise level. In large-noise cases, the truncation approach might occur at near recurrences [10].

We then perform the following computations for each pseudo coordinate $D^{m a} x(t), m=$ $1,2, \ldots, d-1$, of the reconstructed phase space:

1. In the frequency domain, compute $D^{m a} X_{n}=\left(i \omega_{n}\right)^{m a} X_{n}$

2. Invert the FFT to obtain $\hat{y}_{m a}\left(t_{n}\right)=D^{m a} x\left(t_{n}\right)$

3. Retain the $\hat{y}_{m a}\left(t_{n}\right)$ for the interior range of samples for which $\delta\left(t_{n}\right)<\epsilon$ (based on step 3 above).

4. We then normalize each axis of the data, such that $y_{m a}\left(t_{n}\right)=\hat{y}_{m a}\left(t_{n}\right) / R_{m}$, where $R_{m}=$ $\max _{n}\left(\hat{y}_{m a}\left(t_{n}\right)\right)-\min _{n}\left(\hat{y}_{m a}\left(t_{n}\right)\right)$ is the span of the unnormalized coordinate $\hat{y}_{m a}\left(t_{n}\right)$.

Then the $d$-dimensional reconstructed phase space vectors have the form

$$
\begin{aligned}
\mathbf{y}_{n} & =\left[y_{0}\left(t_{n}\right), y_{1}\left(t_{n}\right), y_{2}\left(t_{n}\right), \ldots, y_{d-1}\left(t_{n}\right)\right] \\
& =\left[\frac{x\left(t_{n}\right)}{R_{0}}, \frac{D^{a} x\left(t_{n}\right)}{R_{1}}, \frac{D^{2 a} x\left(t_{n}\right)}{R_{2}}, \ldots, \frac{D^{(d-1) a} x\left(t_{n}\right)}{R_{d-1}}\right] .
\end{aligned}
$$

The value of $a$ is a reconstruction parameter analogous to the delay index in the method of delays. Parameter $a$ can be chosen with the help of an average-mutual-information computation between $y_{0}\left(t_{n}\right)$ and $y_{1} a x\left(t_{n}\right)$. We seek a balance between a low average mutual information, and a sufficiently low derivative order $a(d-1)$. The value of $d=d_{E}$ can then be determined by using the methods in $[5,6]$.

We will use a derivative order such that several dimensions of the reconstruction will not accumulate a very high derivative, and to compare with results on the Lorenz system [12]. We will evaluate the choice of $a$ using the average mutual information, and we will also determine the embedding dimension $d_{E}$, the correlation dimension, and the recurrence behavior, and compare the results between the fractional derivative and delay reconstructions. These quantities have seldom been computed in an embedding not created by the method 
of delays [10]. However, with the Lorenz system [12], the value of $a \approx 0.75$ produced the first minimum of the average mutual information, outperforming integer derivatives. The fractional derivative reconstruction was able to unfold the data effectively, but correlation dimensions were high as the attractor foliation was visibly magnified in the reconstruction [12]. We will compare these trends with the examples presented here.

One more note. The integral definition (2) implies that fractional derivatives, like integrals, have history dependence. Whether this produces temporal distortions in the reconstruction could be an item of investigation.

\section{Examples}

In this section, we reconstruct the phase space for the Duffing equation and data from a two-well-potential experiment. In each example, the phase space is to be reconstructed by means of fractional derivatives, and, for comparison and assessment of the reconstruction parameters, by means of the delay method.

\subsection{Duffing Equation}

The chaotic dynamics of the Duffing equation

$$
\ddot{x}+\alpha \dot{x}+\beta x+\gamma x^{3}=f \cos \omega t
$$

have been thoroughly studied (e.g. [23-25]). We chose parameter values $\alpha=0.2, \beta=\gamma=$ $1, f=27$, and $\omega=1.333$, for which the system has been used as a model for nonlinear string vibrations [26], and studied subsequently for system identification [27]. Since $\beta>0$, this representation of Duffing's equation does not have a two-well potential.

The simulation generated 57344 data with a sampling interval of $\Delta t=0.05$ (the driving period is 4.7136).

In performing the fractional derivative reconstruction, we computed the fractional derivatives, and then removed the leakage effects by truncating 150 sampled points off of each end of the data. This corresponded to a maximum truncation error of $|D x-\dot{x}|<0.0235$ for the remaining time series, where the maximum value of $|\dot{x}|$ was about 13.9 . The characteristic is similar to the plot shown in [12] on the Lorenz system.

\subsubsection{Average Mutual Information}

For evaluating the choice of derivative order $a$, we looked at the average mutual information $\bar{I}$ between $x$ and $D^{a} x$ as a function of $a$ (upper graph of Figure 1). The first minimum in the plot indicates that optimally independent coordinates for $x$ and $D^{a} x$ correspond to the case of $a=1$ : displacement versus velocity. A wide range of values of $a$ provide lower average mutual information with $x$ than the pair $\left(x, D^{2} x\right)$.

For reference, in the method of delays, the sampled observable and its delay are $x_{n}$ and $x_{n+h}$. We plotted the average mutual information between the delay coordinates, as a function of $h$, as shown in the lower plot of Figure 1. 

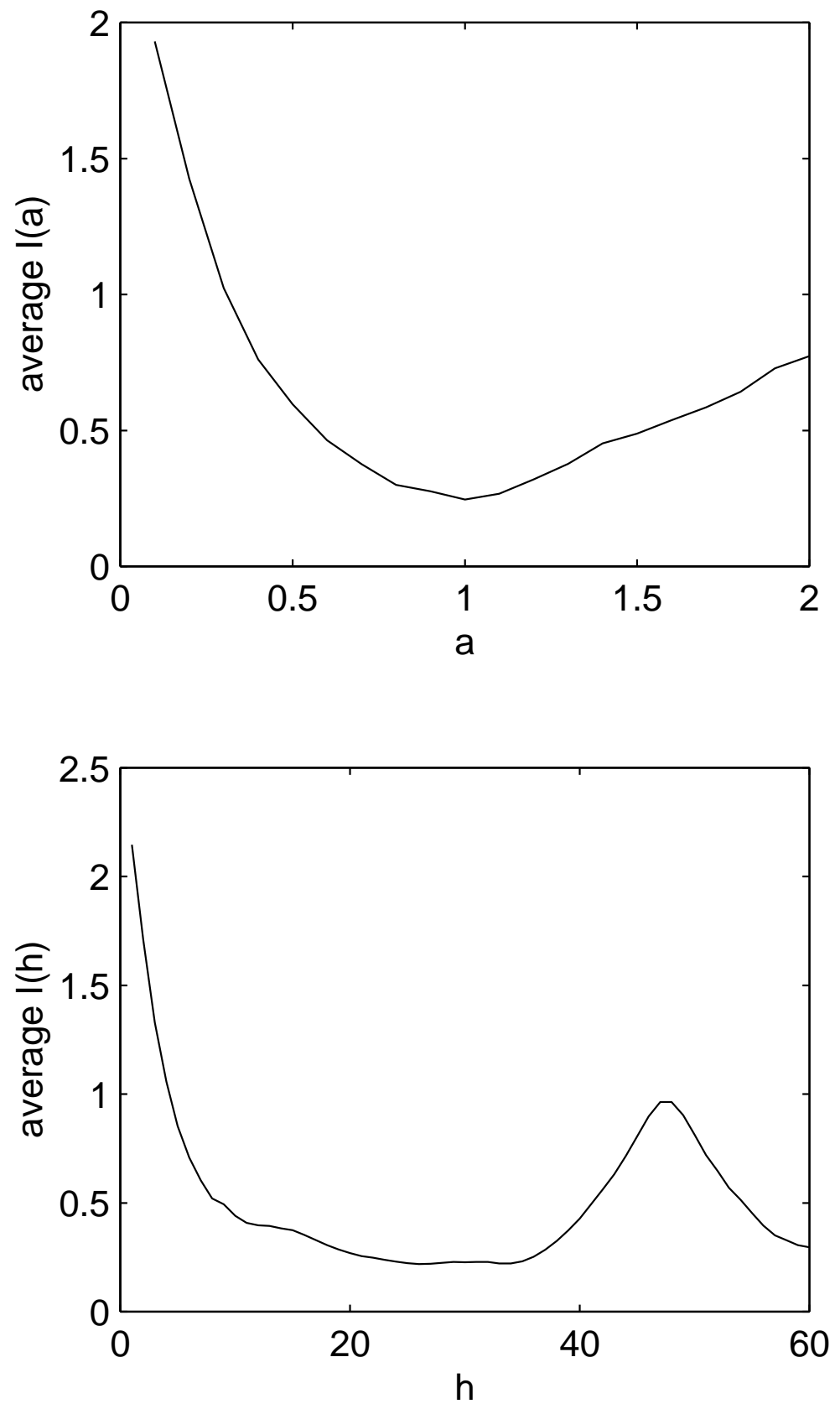

Figure 1: The upper graph shows the average mutual information between the coordinates $x$ and $D^{a} x$ as a function of $a$. The lower plot shows the average mutual information between delay coordinates $x_{n}$ and $x_{n+h}$ as a function of $h$. 
According to the first-local-minimum criterion this plot suggests a delay reconstruction with a delay index of about $h=12$ samples (if there is actually a local minimum there) or $h=25$ samples. (The value of $h=23.6$ corresponds to a quarter period.) The value of $\bar{I}$ at $h=25$ is similar to the average mutual information between $x$ and $D x$. The delay reconstruction would include coordinates with delay indices $m h$, for low integers $m$, whose average mutual information would have non-optimal values. For example, if $h=12$ were chosen as the delay, then non-optimal delays $2 h=24,3 h=36$, and possibly $4 h=48$ would be involved. The well of the minimum is rather wide, suggesting robustness in $h$ for the average mutual information, a bit more so in the delay reconstruction than in the fractional derivative reconstruction. The trend in the Lorenz system for the average mutual information was the opposite, showing lower average mutual information for a broader range of $a$ than $h[12]$.

To obtain several reconstruction coordinates without letting the average mutual information get "large," and without letting the total order of the derivative get "large" so that the noise amplification is reasonable, the choice of fractional derivative orders at multiples of $a=2 / 7$, for illustration of its use for phase-space reconstructions is reasonable. For this value of $a$, the average mutual information $\bar{I}$ is slightly above one. A delay of $h=4$ gives about the same average mutual information between delay coordinates.

\subsubsection{Fractional-Derivative Phase-Space Reconstruction}

Figure 2 shows three fractional derivatives of the signal $x$ from the Duffing system plotted against $x$, and also the plot of $D^{2} x$ vs. $x$ for noise reference.
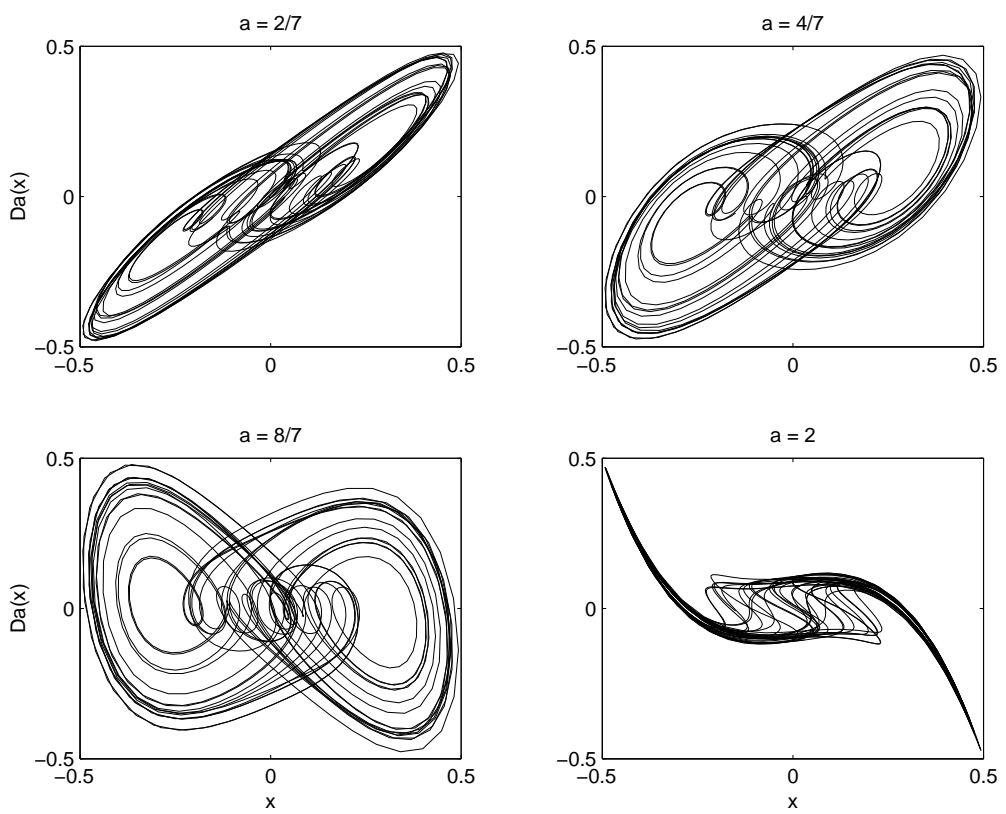

Figure 2: Plots of $D^{a} x$ versus $x$ for various values of $a$ for the Duffing oscillator. 
For visual comparison, Figure 3 shows plots of $x_{n+h}$ versus $x_{n}$. The delay of $h=4$ is visually acceptable.
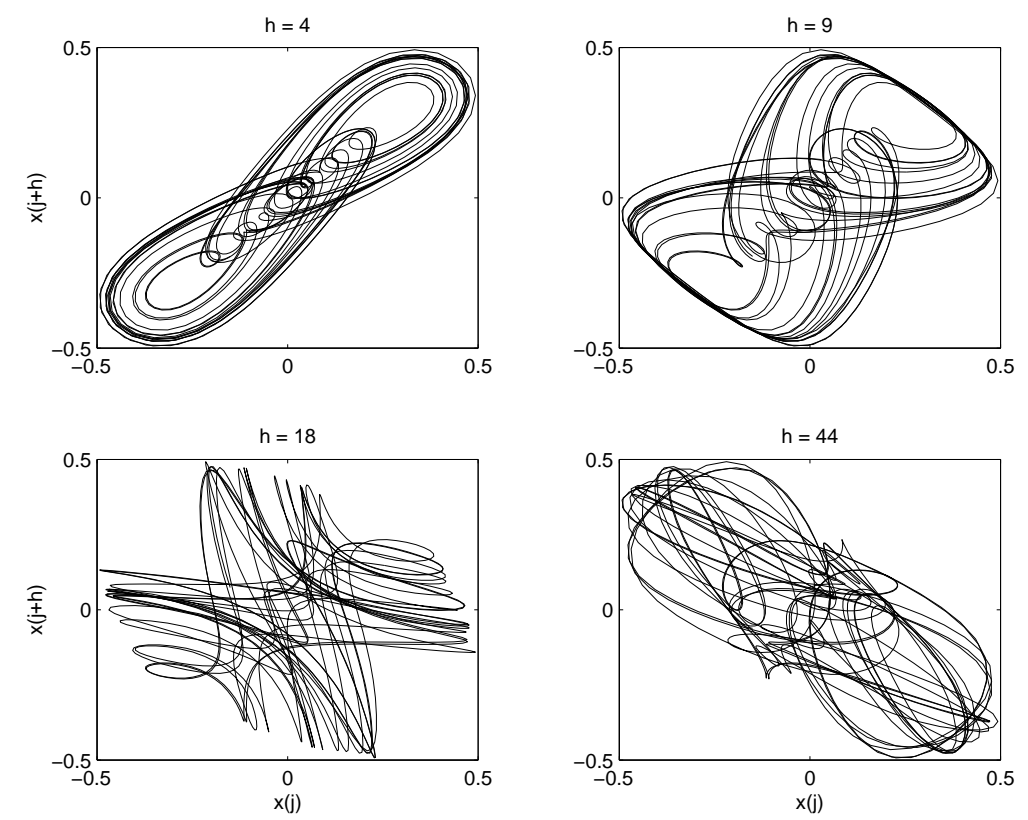

Figure 3: Plots of $x_{n+h}$ versus $x_{n}$ for various values of $h$.

To determine the size of the phase space, we applied the method of global false nearest neighbors (FNNs) [5]. For fractional-derivative reconstructions of the Duffing variable $x$ with orders of multiples of $a=2 / 7$, we found the numbers of false nearest neighbors were 56433, $24819,1162,15,0,0,0$, and 0, for reconstruction dimensions of one through eight. Since there are few FNNs at dimension four, and none at dimension five, we would be inclined to choose four or five as the reconstruction dimension. It is reasonable to expect a dimension of four or more, since we are embedding data generated in a space $\mathbf{R}^{2} \times \mathbf{S}^{1}$ into $\mathbf{R}^{d_{E}}$, where $\mathbf{S}^{1}$ itself embeds into $\mathbf{R}^{2}$.

For the case of delay reconstructions with $h=4$ (the first pair of delay coordinates have about the same average mutual information as the first pair of fractional derivative coordinates), a "legal" choice of $h$, but not optimal based on the average mutual information strategy, the numbers of false nearest neighbors were 56905, 21193, 1272, 0, 0, 0, 0, and 0 for reconstruction dimensions of one through eight. Since there are no false nearest neighbors at dimension four, we can choose an embedding dimension $d_{E}=4$ to fully unfold the data.

Thus, the fractional derivative method unfolded the data with similar efficiency as the method of delays, and is thus considered to be effective.

\subsubsection{Characterization of the Data}

The purpose of performing a phase-space reconstruction is to enable the researcher to effectively characterize the dynamical system, and to enable steps toward system identification 
or nonlinear prediction. Thus, we would like to see if the fractional derivative reconstruction leads to reasonable characterizations of the data.

First we sought recurrences used for extracting unstable periodic orbits (UPOs) [26-31]. Recurrences are defined as instances for which $\left|\mathbf{y}_{n}-\mathbf{y}_{n+k}\right|<\epsilon$, where $\mathbf{y}_{m}$ is the reconstructed vector, and $\epsilon$ is a tolerance chosen by prescription. $\epsilon$ is chosen to be small enough with respect to the size of the attracting set such that it represents a near return of the trajectory, but must be large enough that a practical number of recurrences are identified. It may often be in the range of $0.5-1.5 \%$ of the span of the data.

A recurrence plot from the four-dimensional fractional-derivative reconstruction for the Duffing system, shown as the solid line in Figure 4, indicates the numbers of recurrences at various multiples of the driving period. For comparison, the recurrence plot of the true phase space is shown in the same figure. The recurrence plot shows that relatively similar numbers of like-period orbits are found in both the reconstructed and the true phase spaces. The true phase space reveals slightly more recurrences. Comparison of the recurrence indices indicate that when a recurrence is found in both spaces, it occurs at nearly the same index. Hence, the fractional-derivative leads to little distortion in the temporal location of recurrences.

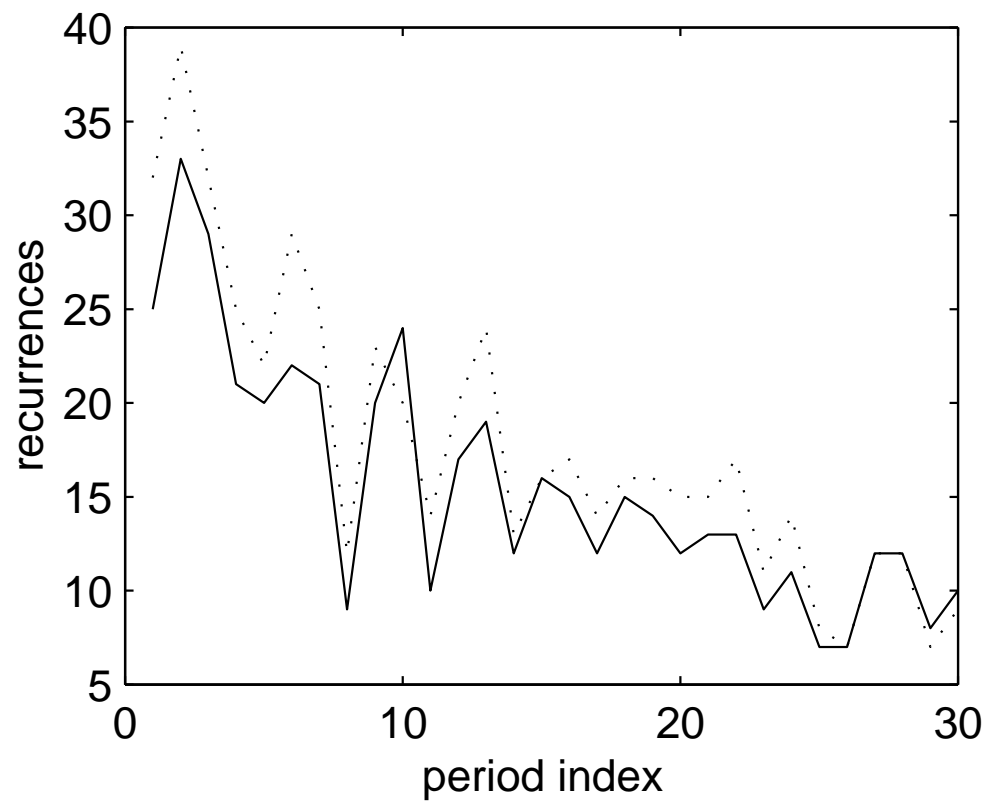

Figure 4: A recurrence plot of data from the Duffing oscillator. The solid line is from the fractional derivative reconstruction, and the dotted line is from the true phase space.

Finally, we computed fractal dimensions of the reconstructed and true data sets. The dimensions were computed in the Poincaré section, obtained transverse to the $\mathbf{M}^{d}=\mathbf{R}^{(d-1)} \times$ $\mathbf{S}^{1}$ space by sampling at a fixed phase of excitation. (The assumption is that $\mathbf{M}^{d}$ has similar FNN characteristics as the reconstructed $\mathbf{R}^{d}$ space.) We computed the limit capacity (box dimension) $D_{0}$, the information dimension $D_{1}$, and the correlation dimension $D_{2}$, using a fast box-covering algorithm [32,33], and we also determined the correlation dimension, $D_{c}$, 
by computing the slower correlation integral $C(r)$ for various ball sizes, $r[34,35]$. The values of the $D_{i}$ were computed over a three-decade range of ball sizes $r$ (30 points equally spaced in $\log _{2}(r)$ ) which produced a nearly linear scaling region, while $D_{c}$ was estimated using a linear scaling region covered by about about five logarithmically spaced correlation integral data over three box-size doublings. Both dimension estimate methods are based on the slope of a summed quantity $N$ (e.g. number of boxes, partition sum, or correlation sum) plotted against the log of the box size. Errors in the slope estimates are based on $95 \%$ confidence intervals using the ideas in Beck and Arnold [36]. The error in the dimension estimate is based on assumptions requiring additive, zero mean, constant variance, and uncorrelated error in the sum $N$, as well as zero error in the box size. (While we are unable to check these assumptions, they are probably reasonable for a computed sum.)

For the true phase space, we used a two-dimensional Poincaré section and obtained $D_{0}=1.39 \pm 0.06, D_{1}=1.40 \pm 0.05$, and $D_{2}=1.38 \pm 0.05$. The correlation dimension was estimated as $D_{c}=1.24 \pm 0.02$. The dimension of the fractional derivative reconstruction was obtained from three-dimensional Poincaré section data (consistent with the FNN results), yielding $D_{0}=1.42 \pm 0.06, D_{1}=1.50 \pm 0.08$, and $D_{2}=1.46 \pm 0.10$. We note that $D_{1}$ should be lower than $D_{0}[37,38]$. The computations are based on the slope of the box-counted partition function, which can be sensitive. Furthermore, in box-counting, the information dimension is treated as a singular case in the computation [32,37], which could lead to a small systematic error. The dimension by correlation integral was $D_{c}=1.32 \pm 0.04$. The dimension of the delay reconstruction phase space was obtained from three-dimensional Poincaré section data as $D_{0}=1.48 \pm 0.06, D_{1}=1.38 \pm 0.05$, and $D_{2}=1.31 \pm 0.06$. The correlation dimension was $D_{c}=1.26 \pm 0.06$.

The dimension estimates for the fractional derivative reconstruction of the Duffing system was more consistent than as seen in the Lorenz system [12].

\subsubsection{Comments on Noise}

To get a brief glimpse of the effect of noise, we consider the case of additive noise, such that the noisy signal is $z(t)=x(t)+n(t)$, where $x(t)$ is the true signal and $n(t)$ is the noise. Then the fractional derivative is $D^{a} z(t)=D^{a} x(t)+D^{a} n(t)$. As the Fourier transform is a linear operation, the effect of noise on $D^{a} z(t)$ scales with the level of noise $n(t)$. We have added random noise to each sample of the Duffing displacement data. The random noise was uniformly distributed in the range $[-\epsilon, \epsilon]$, where $\epsilon=0.01\left(x_{\max }-x_{\min }\right.$, that is $1 \%$ of the span of the displacement data. The data $n(t)$ was generated using the 'rand' function in Matlab. As such, $n(t)$ had a mean squared value of $\epsilon / 3$. Figure 5 shows the normalized error in $D^{a} z(t)$, i.e. the ratio of the root mean squared value of $\left(D^{a} z-D^{a} x\right)$ to the root mean squared value of $D^{a} x$, for various values of $a$. At $a=0$ we see the normalized error of $z$. The plot shows an example of how this uniform (high-frequency) noise grows with $a$.

\subsection{An Experimental Two-Well Oscillator}

We now apply the fractional derivative reconstruction to data from a forced magneto- 


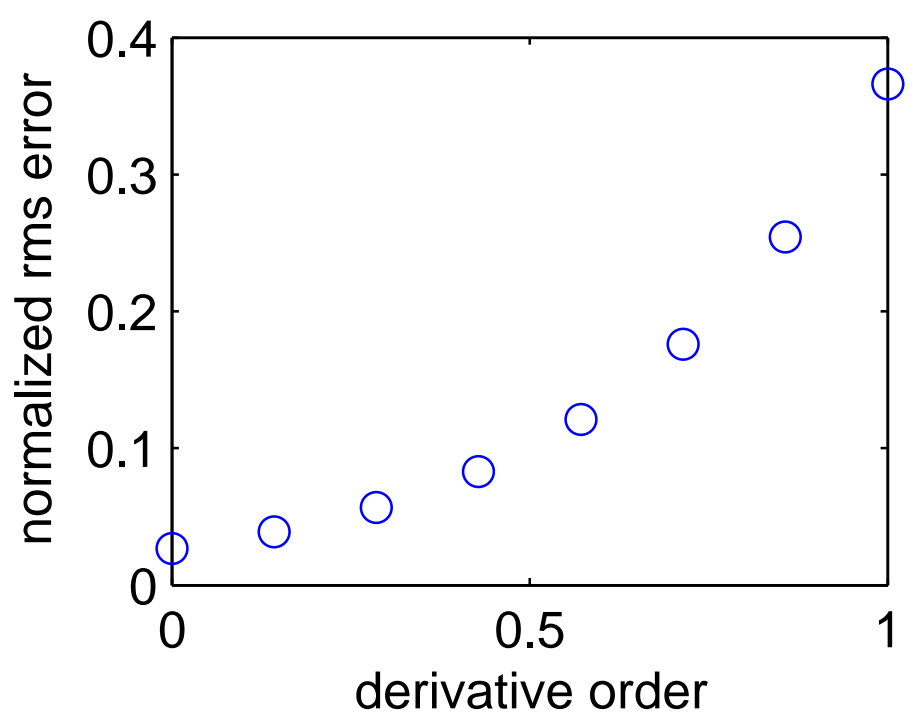

Figure 5: The root mean squared error, of the noisy Duffing data, as the ratio of the root mean squared value of $\left(D^{a} z-D^{a} x\right)$ to the root mean squared value of $D^{a} x$, for various values of $a$.

elastic oscillator that has a two-well potential [39]. A brief description is given below, although a more detailed description can be found in references [40-42].

\subsubsection{Experimental Setup}

The experiment consisted of a stiffened beam buckled by two magnets. Two rare-earth permanent magnets were placed on the base of the frame holding the beam to create the two-well potential. The beam had extra rigidity in the form of steel bars epoxied and bolted along the length away from the clamped end. This additional rigidity was included to make the system behave as a single degree of freedom. The fruit of this effort includes the recovery of the stable and unstable manifolds by means of stochastic interrogation [40]. The uncovered portion of the beam near the clamped end acted as an elastic hinge from which the position of the beam was measured by strain gauges. The frame was then fixed through a rigid mount to an electromagnetic shaker. A periodic driving signal was fed through a power amplifier to the shaker to provide the external forcing function.

Data from the strain gauges were acquired using a 12-bit, $\pm 5 \mathrm{~V}$ data-acquisition $(\mathrm{A} / \mathrm{D})$ board, with the digital values from -2048 to 2047 corresponding to $-5 \mathrm{~V}$ to $5 \mathrm{~V}$. With no forcing, three equilibria exist: two are stable at digital values of -495 and $315(-1.21 \mathrm{~V}$ and $0.77 \mathrm{~V}$ ), and one is unstable (saddle) at approximately zero. The driving frequency was set at $7.5 \mathrm{~Hz}$ with $1.5 \mathrm{~V}$ of the function generator output, by which chaotic data were generated, passed through a $50 \mathrm{~Hz}$ low-pass filter, and collected at the sampling frequency of $187.5 \mathrm{~Hz}$. 
At this sampling frequency and driving frequency, there are 25 samples per driving period. A total of 57344 data were collected.

After computing the fractional derivatives, we truncated 150 points from both ends of the time series data. The trend of the error $|D x-\dot{x}|$ was similar to the Duffing system.

\subsubsection{Average Mutual Information}

The optimal value of the average mutual information for the fractional-derivative pair is less than 0.1, occurring at $a=1$ (displacement vs. velocity), according to the upper plot in Figure 6. In order to obtain several fractional derivative coordinates, while keeping the largest derivative less than two, we again use multiples of $a=2 / 7$, for which the average mutual information is slightly above one.

The first local minimum of the average mutual information between delay coordinates occurs at a delay of $h=8$, with $\bar{I} \approx 0.6$ (Figure 6 , lower plot). For a delay of $h=4$, the average mutual information between the delay pair is similar to the average mutual information between the fractional-derivative pair with $a=2 / 7$.

In this case, the average mutual information was more robustly low in value with respect to $a$ than to $h$ in the vicinity of its first minimum, although lower for $h$ than $a$ for larger values.

\subsubsection{Reconstruction of the Phase Space}

Figure 7 shows pseudo phase portraits involving three fractional derivatives of the signal $x$ from the two-well oscillator plotted against $x$, and also the plot of $D^{2} x$ vs. $x$ for noise reference. In this case, $D^{2}$ is considerably noisy, and we probably would not want reconstruction coordinates with a fractional derivative approaching this order. For comparison, the delay-space plots of the two-well data are shown in Figure 8. The values of $h$ shown provide coordinate combinations for reconstructions based on $h=4$ (which has a comparable average mutual information with that of the fractional derivative with $a=2 / 7$ ), and based on $h=8$, for which the average mutual information was minimum in the delay coordinates.

For fractional-derivative reconstructions of the two-well variable $x$ with orders of multiples of $a=2 / 7$, we found the numbers of false nearest neighbors were 56461,32032, 396, 8, $0,0,0$, and 0 , for reconstruction dimensions of one through eight. Since there are few FNNs at dimension four and none for five and above, we would be inclined to choose $d_{E}=4$ or $d_{E}=5$ as the reconstruction dimension.

For the case of delay reconstructions with $h=4$, for which the average mutual information matched that of the fractional derivative pair with $a=2 / 7$, the numbers of false nearest neighbors were 56952,31999, 4103, 6, 2, 2, 0 , and 0 for reconstruction dimensions of one through eight. We might choose an embedding dimension in the range of $d_{E}=4$ or 5 to represent the data, if we assume that the low numbers of false nearest neighbors will not affect calculations in the reconstruction space.

Thus, the ability of the fractional derivative method to unfold the data with increments 

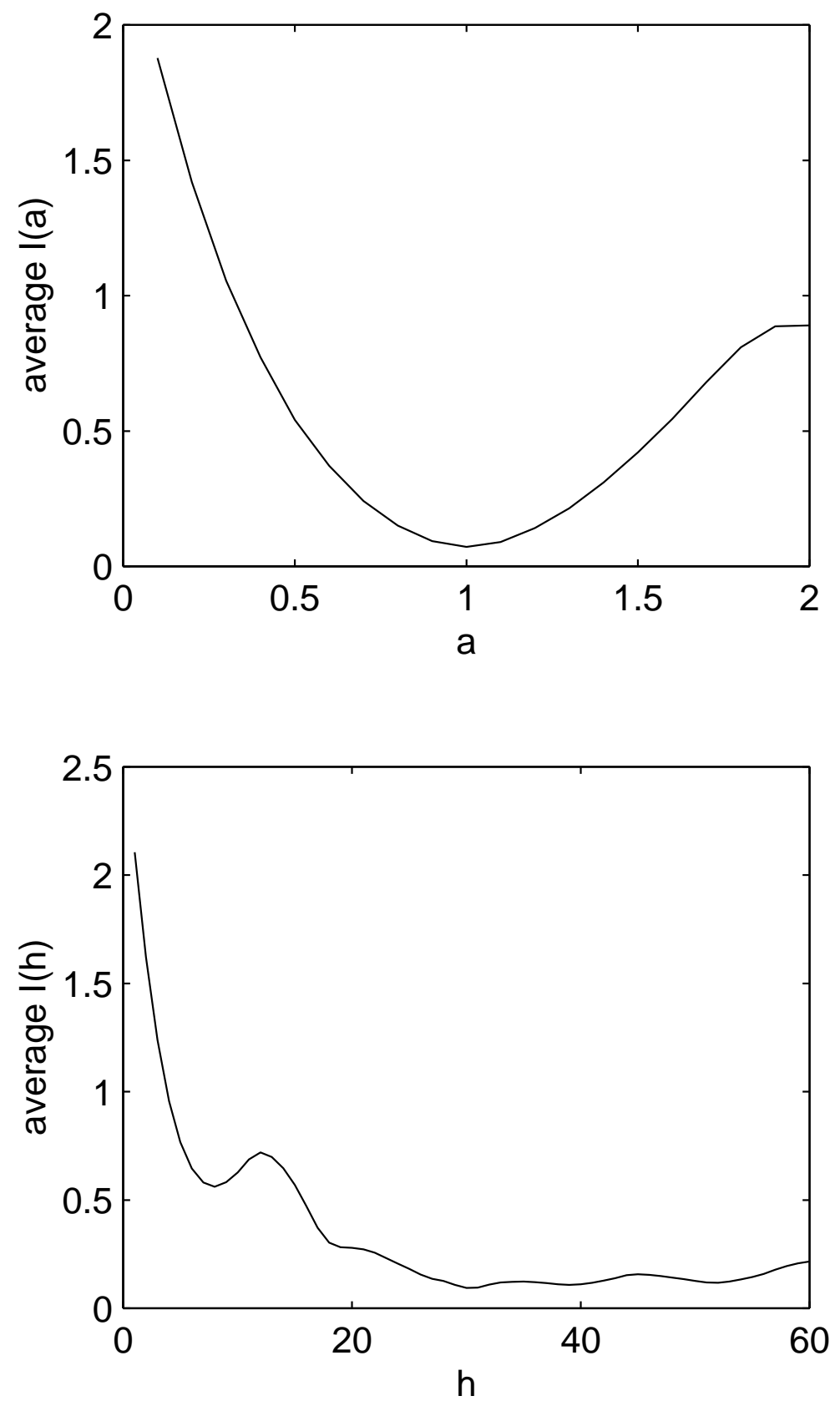

Figure 6: The upper graph shows the average mutual information between the coordinates $x$ and $D^{a} x$ as a function of $a$ for the two-well experiment. The lower plot shows the average mutual information between delay coordinates $x_{n}$ and $x_{n+h}$ as a function of $h$. 

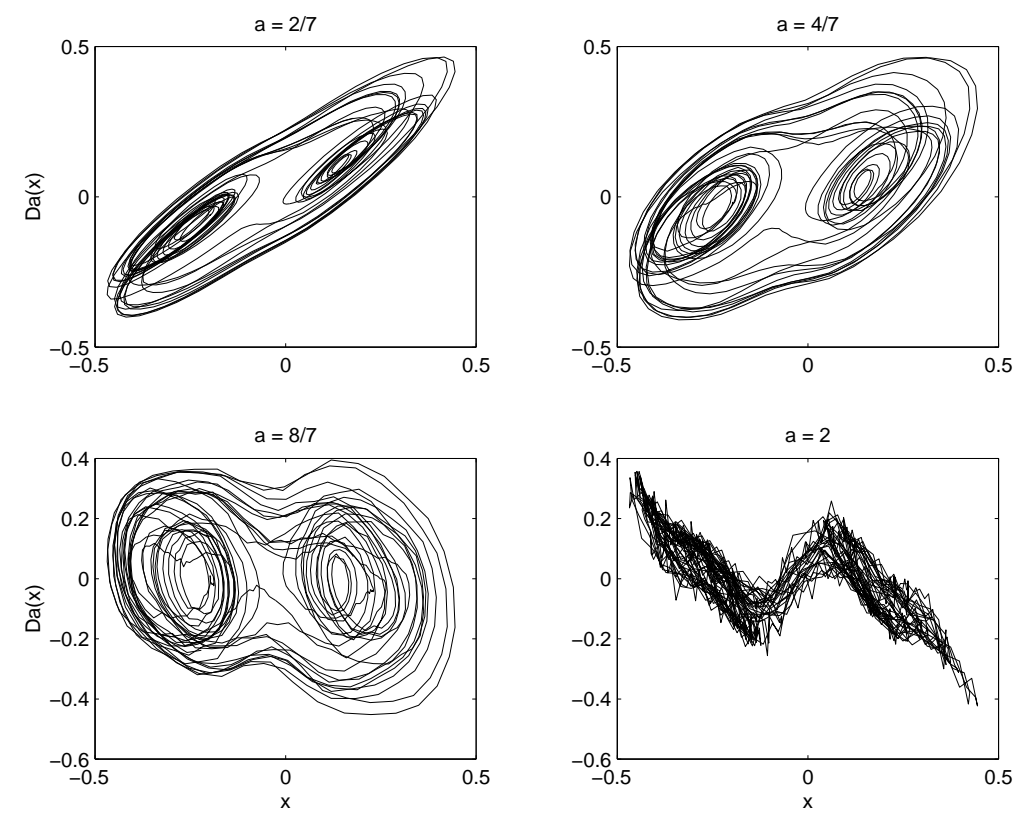

Figure 7: Plots of $D^{a}(x)$ versus $x$ for various values of $a$ for the two-well experiment.
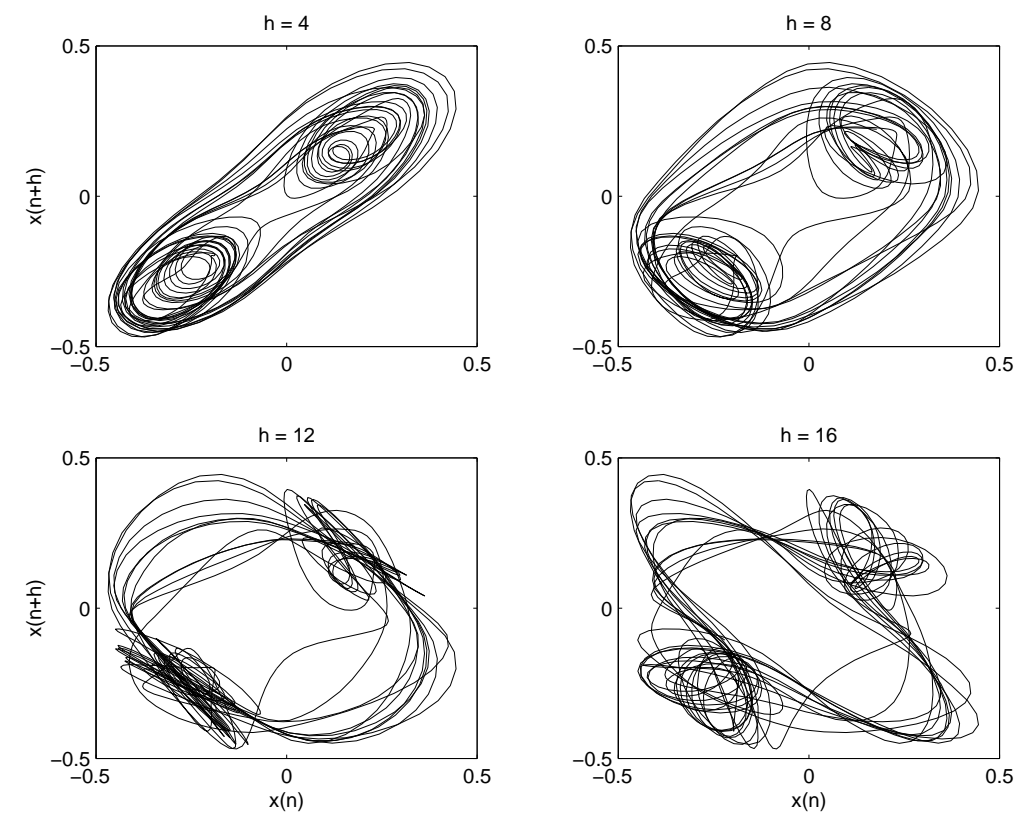

Figure 8: Plots of $x_{n+h}$ versus $x_{n}$ for various values of $h$ for the two-well experiment. 
of $a=2 / 7$ is similar to that of the method of delays with $h=4$. The unfolding of the data is in the range of the expected reconstruction dimension.

\subsubsection{Characterization of the Data}

A recurrence plot from the five-dimensional fractional-derivative reconstruction for the two-well oscillator, shown in Figure 9, indicates the numbers of recurrences at various multiples of the driving period. For comparison, the recurrence plot of the true phase space is shown in the same figure. The recurrence plot shows that relatively similar numbers of likeperiod orbits are found in both the reconstructed and the true phase spaces. Comparison of the recurrence indices indicates that when a recurrence is found in both spaces, it occurs at nearly the same index. Hence, the fractional-derivative leads to little distortion in the temporal location of recurrences, as compared to the true phase space.

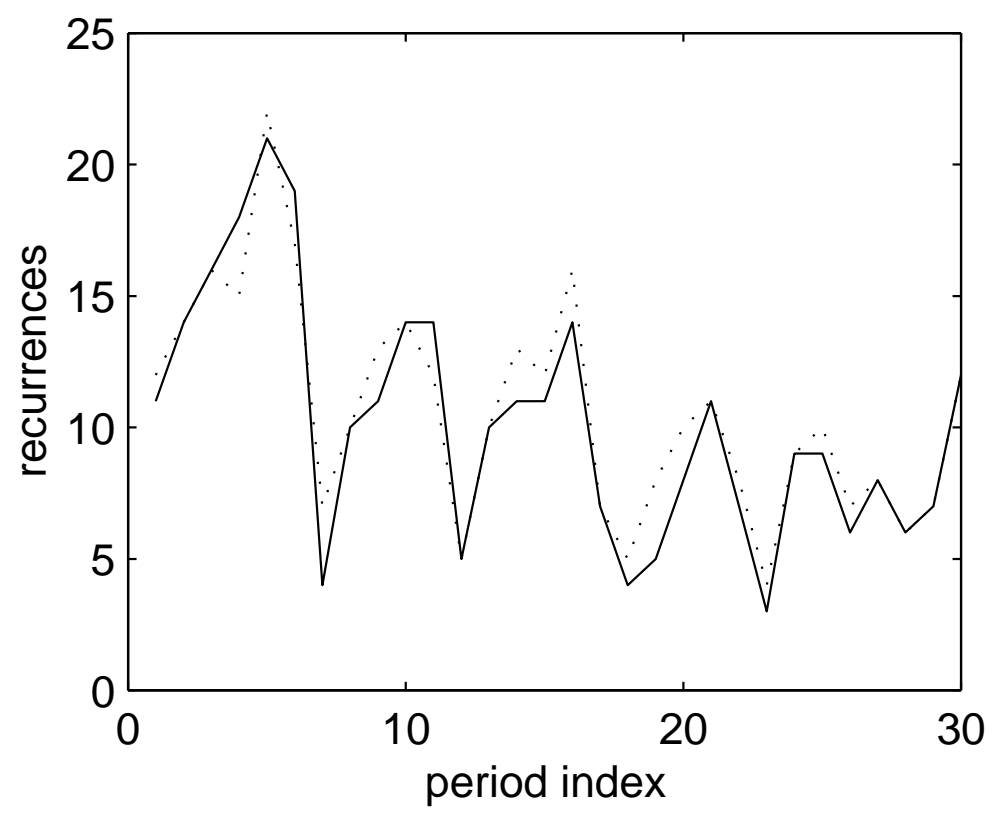

Figure 9: The number of recurrences versus the period index for of data from the twowell experiment. The solid line shows the recurrence plot from the fractional derivative reconstruction, and the dotted line shows the recurrence plot from the true phase space.

The dimensions $D_{0}, D_{1}$, and $D_{2}$ were computed in the constant-phase Poincaré section over two decades of box sizes. For the true phase space in a two-dimensional Poincaré section, we obtained $D_{0}=1.68 \pm 0.04, D_{1}=1.67 \pm 0.02$, and $D_{2}=1.59 \pm 0.03$. For the fractional derivative reconstruction in a three-dimensional phase space (based on FNN results), we had $D_{0}=1.66 \pm 0.04, D_{1}=1.69 \pm 0.03$, and $D_{2}=1.65 \pm 0.06$. For a three-dimensional Poincaré section of the delay reconstruction, $D_{0}=1.62 \pm 0.09, D_{1}=1.65 \pm 0.04$, and $D_{2}=1.58 \pm 0.06$. Again, the information dimension was sometimes larger than the box dimension, indicating some error. 
Thus, the fractional derivative reconstruction led to dimension quantities that were on par with estimates from both other representations of the phase space.

\section{Conclusion}

We have used fractional derivatives as a means of reconstructing the phase space of lowdimensional forced nonlinear oscillators, including an experiment. The order of the fractional derivative is a parameter in the reconstruction, analogous to the delay index in the method of delays. The fractional order was chosen rather qualitatively to minimize the total derivative order, and hence the noise amplification, in the reconstruction coordinates, while examining the the average mutual information to assess the independence between the first coordinate pair. In these forced oscillator examples, the "optimal" value of the derivative order $a$ for minimizing the average mutual information was $a=1$, corresponding to displacement and velocity. This is not the rule, as the Lorenz system was seen to optimize at $a \approx 0.75$ [12]. Also, the fractional derivative and the delay reconstructions were comparable in their ability to minimize the average mutual information, and thereby obtain independent coordinates in the reconstruction.

The fractional derivatives successfully unfolded the phase space in the forced oscillators, based on the FNN test applied to the reconstructed data. The resulting embedding dimensions were within one dimension of the result for delay embeddings of the same systems, with the delay chosen to produce the same average mutual information.

Further characterizations of the dynamics were similar for the various representations of the phase space. The extraction of unstable periodic orbits from the fractional-derivative pseudo phase space and the true phase space produced similar recurrence plots. Measures of the fractal dimensions were computed in the fractional derivative reconstructions, the delay reconstructions, and the true phase spaces. The box, information, and correlation dimensions were consistent among the phase spaces for these examples.

The fractional derivative method is simple, requiring simple Matlab code, for example. Of course, the method of delays is more simple, and is expected to continue as the preferred method, except in special situations. History dependence may make it applicable to nonsmooth problems [11]. For example, a stick-slip trajectory causes a collapse in the reconstructed phase space [43]. The fractional derivative can hold memory, and likely prevent this collapse. The method is also interesting from the point of view of the increasing awareness and applications of fractional derivatives. The successful application to low-noise experimental data shows feasibility with real data.

While fractional derivatives have produced useful phase space reconstructions in these examples, there is yet no mathematical proof of the ability of fractional derivative reconstructions to perform embeddings.

\section{Acknowledgements}

We are thankful to our sponsors: the NASA-Langley Research Center (grant number NAG-1-01048), with Dr. Walt Silva; and the National Science Foundation (GOALI grant 
number DMII-9800323). Any opinions, findings, and conclusions or recommendations expressed in this material are those of the author and do not necessarily reflect the views of the National Science Foundation. We are very grateful to Prof. Joe Cusumano of Penn State University for providing experimental data from a two-well magneto-elastic oscillator, and to Prof. James Beck of Michigan State University for discussions on parameter estimation.

6. *

References

[1] Takens, F., "Detecting strange attractors in turbulence," Lecture Notes in Mathematics 898, Springer, 366-381 (1981).

[2] Packard, N. H., Crutchfield, J. P., Farmer, J. D., and Shaw, R. S., "Geometry from a time series," Physical Review Letters 45 (9) 712-716 (1980).

[3] Noakes, L., "The Takens Embedding Theorem," International Journal of Bifurcation and Chaos 1 (4) 867-872 (1991).

[4] A. M. Fraser and H. L. Swinney, "Independent coordinates for strange attractors from mutual information," Physical Review A 33 (2), 1134-1140 (1986).

[5] Kennel, M., Brown, R., and Abarbanel, H. D. I., "Determining embedding dimension for phase-space reconstruction using a geometrical construction," Physical Review A 45, 3403-3411 (1992).

[6] Cao, L., "Practical Method for Determining the Minimum Embedding Dimesino of a Scalar Time Series," Physica D 110 43-50 (1997).

[7] Potopov, A., "Distortions of Reconstructeion for Chaotic Attractors," Physica D 101 207-226 (1997).

[8] Mindlin, G. B., and Solari, H. G., "Topologically Inequivalent Embeddings," Physical Review E 52 (2) 1497-1502 (1995).

[9] Gilmore, R., "Topological Analysis of Chaotic Dynamical Systems," Review of Modern Physics 70 (4) 1455-1526 (1998).

[10] Gilmore, R. and Lefranc, M., The Topology of Chaos, Wiley-Interscience, New York (2002).

[11] Lin, G., Phase-Space Reconstruction by Alternative Methods, M.S. Thesis, Michigan State University, East Lansing (2001).

[12] Feeny, B. F. and Lin, G., Fractional Derivatives Applied to Phase Space Reconstructions, Nonlinear Dynamics 38 (1-4) 85-99 (2004). Special Issue: Fractional Derivatives and Their Applications, O. P. Agrawal, J. A. Tenreiro Machado, and J. Sabatier (editors). 
[13] Bagley, R. L. and Calico, R. A., "Fractional Order State Equations for the Control of Viscoelastically Damped Structures," AIAA Journal of Guidance, 14 (2) 304-311 (1991).

[14] Padovan, J. and Sawicki, J. T., "Nonlinear Vibrations of Fractionally Damped Systems," Nonlinear Dynamics 16 (4) 321-336 (1998).

[15] Zhang, W., and Shimizu, N., "Numerical Algorithm for Dynamic Problems Involving Fractional Operators," JSME International Journal, Series C, 41 (3) 364-370 (1998).

[16] He, J. H., "Approximate Analytical solution for Seepage Flow with Fractional Derivatives in Porous Media," Computational Methods in Applied Mechanics and Engineering 167 57-68 (1998).

[17] Stiassnie, M., "A Look at Fractal Functions through their Fractional Derivatives," Fractals 5 (4) 561-564 (1997).

[18] Machado, J. A. T. (ed.), Nonlinear Dynamics 29 (1-4), (2002). Special Issue of Fractional Order Calculs and its Applications.

[19] Agrawal, O. P., Machado, J. A. T. and Sabatier, J. "Special Issue: Fractional Derivatives and their Applications - Introduction," Nonlinear Dynamics 38 (1-4), 1-2 (2004).

[20] Oldham, K. B. and Spanier, J., The Fractional Calculus. Theory and Application of Differentiation and Integration to Arbitrary Order, Academic Press, New York and London (1974).

[21] Tseng, C.-C., Pei, S.-C., Hsia, S.-C., "Computation of Fractional Derivatives using Fourier Transform and Digital FIR Differentiator," Signal Processing 80 151-159 (2000).

[22] Ewins, D. J., 1984, Modal Testing: Theory and Practice, Research Studies Press, Letchworth, Hertfordshire, England.

[23] Ueda, Y., The Road to Chaos, Aerial Press (1994).

[24] Guckenheimer, J., and Holmes, P. J., Nonlinear Oscillations, Dynamical Systems, and Bifurcations of Vector Fields, Springer-Verlag, New York (1983).

[25] Moon, F. C., Chaotic and Fractal Dynamics-An Introduction for Applied Scientists and Engineers, John Wiley and Sons, New York (1992).

[26] N. B. Tufillaro, T. Abbott, and J. Reilly, An Experimental Approach to Nonlinear Dynamics and Chaos, Addison-Wesley (1992).

[27] C.-M. Yuan and B. F. Feeny, "Parametric identification of chaotic systems," Journal of Vibration and Control 4 (4), 405-426 (1998).

[28] D. Auerbach, P. Cvitanovic, J.-P. Eckmann, G. Gunaratne, and I. Procaccia, "Exploring chaotic motion through periodic orbits," Physical Review Letters 58, 2387-2389 (1987). 
[29] D. P. Lathrop and E. J. Kostelich, "Characterization of an experimental strange attractor by periodic orbits," Physical Review A 40, 4028-4031 (1989).

[30] Van de Water, W., Hoppenbrouwers, M., and Christiansen, F., "Unstable Periodic Orbits in the Parametrically Excited Pendulum," Physical Review A 44 (10) 6388-6398 (1991).

[31] Van de Wouw, N., Verbeek, G., and Van Campen, D. H., "Nonlinear parametric identification using chaotic data," Journal of Vibration and Control 1, 291-305 (1995).

[32] Feeny, B. F., "Fast multi-fractal analysis by recursive box covering," International Journal of Bifurcation and Chaos 10 (9) 2277-2287 (2000).

[33] Molteno, T. C. A., "Fast $O(N)$ box-counting algorithm for estimating dimensions," Physical Review E48 (5) R3263-R3266 (1993).

[34] Grassberger, P., and Proccacia, I., "Characterization of Strange Attractors," Physical Review Letters 50 346-349 (1983).

[35] Malraison, G., Atten, P., Berge, P., and Dubois, M., "Dimension of Strange Attractors: An Experimental Determination of the Chaotic Regime of Two Convective Systems," Journal of Physics Letters 44 897-902 (1983).

[36] Beck, J. V. and Arnold, K. J., Parameter Estimation in Engineering and Science, Wiley, New York (1977).

[37] Feder, J., Fractals, Plenum Press, New York.

[38] Gerschenfeld, N., 1988, “An Experimentalist's Introduction to the Observation of Dynamical Systems," in Directions in Chaos, Vol. II, Hao Bai-Lin (ed.), World Scientific, Singapore (1988).

[39] Cusumano, J. P., The data used for this experiment was generated in the Engineering Science and Mechanics Department, Penn State University, College Station, PA (1994).

[40] J. P. Cusumano and B. Kimble, "Experimental observation of basins of attraction and homoclinic bifurcation in a magneto-mechanical oscillator," Nonlinearity and Chaos in Engineering Dynamics, J. M. T. Thompson and S. R. Bishop, eds., John Wiley and Sons, Chichester, 71-89 (1994).

[41] J. P. Cusumano and B. Kimble, "A stochastic intorrogation method for experimental measurements of global dynamics and basin evolution-application to a 2-well oscillator," Nonlinear Dynamics 8 (2) 213-235 (1994).

[42] Feeny, B. F., Yuan, C. M., and Cusumano, J. P., "Parametric Identification of an Experimental Magneto-Elastic Oscillator," Journal of Sound and Vibration 247 (5) 785-806 (2001). 
[43] Feeny, B. F., and Liang, J.-W., "Phase-Space Reconstructions and Stick-Slip," Nonlinear Dynamics 13 39-57 (1997). 